\title{
Social Choice with Analytic Preferences
}

by

Michel Le Breton and John A. Weymark

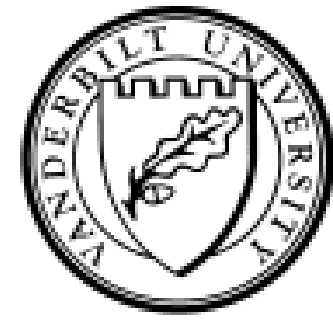

Working Paper No. 00-W23R

June 2000

Revised March 2001

DEPARTMENT OF ECONOMICS

VANDERBILT UNIVERSITY

NASHVILLE, TN 37235

www.vanderbilt.edu/econ 


\title{
Social Choice with Analytic Preferences*
}

\section{Michel Le Breton ${ }^{1}$, John A. Weymark ${ }^{2}$}

${ }^{1}$ GREQAM, Université de la Méditérranée, Chateau Lafarge, Route des Milles, 13290

Les Milles, France

(e-mail: lebreton@romarin.univ-aix.fr)

${ }^{2}$ Department of Economics, Box 1819, Station B, Vanderbilt University, Nashville, TN 37235, USA

(e-mail: john.weymark@vanderbilt.edu)

Revised, March 2001

Address for Proofs. John A. Weymark, Department of Economics, Box 1819, Station B, Vanderbilt University, Nashville, TN 37235, USA. Fax: 1-615-343-8495.

Proposed Running Head. Social choice with analytic preferences

\begin{abstract}
Arrow's axioms for social welfare functions are shown to be inconsistent when the set of alternatives is the nonnegative orthant in a multidimensional Euclidean space and preferences are assumed to be either the set of analytic classical economic preferences or the set of Euclidean spatial preferences. When either of these preference domains is combined with an agenda domain consisting of compact sets with nonempty interiors, strengthened versions of the Arrovian social choice correspondence axioms are shown to be consistent. To help establish the economic possibility theorem, an ordinal version of the Analytic Continuation Principle is developed.
\end{abstract}

Journal of Economic Literature classification number. D71

*We are grateful to Walter Bossert, Mark Johnson, Steve Slutsky, Yves Sprumont, our referees, and an editor of this journal for their comments. This research was supported in part by a grant from the Social Sciences and Humanities Research Council of Canada. 


\section{Introduction}

A social welfare function is a mapping from a set of profiles of individual preference orderings to the set of social orderings on a universal set of alternatives. Arrow's (1963) Theorem shows that it is impossible for a social welfare function to satisfy Independence of Irrelevant Alternatives (the social ranking of a pair of alternatives only depends on the individual rankings of these alternatives), Weak Pareto (if everyone strictly prefers one alternative to a second, then so does society), and Nondictatorship (nobody's strict preferences are always respected) if the domain of preference profiles is unrestricted and there are at least three alternatives being ranked.

Building on the work of Kalai, Muller, and Satterthwaite (1979), a number of articles have investigated the consistency of Arrow's axioms when natural economic or political restrictions are placed on the universal set of alternatives and on the domain of preference profiles. Le Breton and Weymark (1996, 2001) and Le Breton (1997) provide introductions to this literature. The general conclusions to emerge from this line of research are that when the set of alternatives is multidimensional, unless the domain is exceedingly small, either Arrow's axioms are inconsistent or there is an individual who is dictatorial over most pairs of alternatives. ${ }^{1}$ Thus, simply modifying the domain of the social welfare function does not seem to be a promising way to escape from Arrow's dilemma when the set of alternatives is multidimensional.

Arrow's Theorem can also be formulated in terms of a social choice correspondence. In this case, the objective is to choose a set of socially optimal alternatives from a feasible subset of the alternatives called an agenda. More precisely, a social choice correspondence specifies a nonempty subset of the agenda for each admissible preference profile and each admissible agenda. In its choice-theoretic formulation, Arrow's Theorem shows that Arrow's Choice Axiom (the choice-theoretic analogue of Arrow's assumption that social preferences are orderings), Independence of Infeasible Alternatives (the social choice only depends on the preferences for feasible alternatives), and the choice correspondence versions of Weak Pareto (only weakly Pareto optimal alternatives are chosen) and Nondictatorship (the chosen alternatives are not always a subset of one individual's best feasible alternatives) are inconsistent if the preference domain is unrestricted and the agenda domain includes all the two and three element subsets of the universal set.

The requirement that some of the agendas are finite is unnatural in many economic problems. For example, an agenda could be an Edgeworth box in an exchange economy or, alternatively, all of the allocations that are feasible for a production economy with given production technologies and resource endowment. By varying the technologies and/or the endowment, new agendas are obtained. The literature on Arrovian social choice correspondences on restricted domains is surveyed in Le Breton (1997) and Le Breton and Weymark (2001).

In this article, we establish two possibility theorems for social choice correspondences on restricted domains. In our theorems, the set of alternatives is the non-

\footnotetext{
${ }^{1}$ For a one-dimensional set of alternatives, the majority rule social welfare function satisfies Arrow's axioms on the domain of single-peaked preferences if there are are an odd number of individuals. See Black (1948) for a discussion of majority rule with single-peaked preferences.
} 
negative orthant of a Euclidean space of dimension at least two. We assume that the agenda domain only contains compact sets with nonempty interiors, as in the economic examples given above. It is also natural to suppose that the agenda is a nonfinite compact set when a legislature is simultaneously determining the values of a number of issues, such as the budgets of different bureaus. For the preference domain, we consider two alternative domains of analytic preference profiles. A preference is analytic if it can be represented by an analytic utility function; i.e., by a function that can be expanded locally at each point in its domain as a Taylor series. Polynomials are examples of analytic functions.

In our first possibility theorem, the preference domain contains only Euclidean spatial preferences. With a Euclidean spatial preference, there is an ideal point and the further an alternative is from this alternative (as measured by Euclidean distance), the worse it is. Spatial preferences are commonly used in political models of elections and legislatures. ${ }^{2}$ A Euclidean spatial preference is analytic because it can be represented by a quadratic utility function.

In our second possibility theorem, the preference domain consists solely of preferences that are monotone and that can be represented by an analytic utility function with no critical points. Further restrictions on preferences such as convexity are permitted. A possible motivation for considering monotone analytic preferences is provided by the fact that many of the functional forms used for utility functions in economic models, such as Cobb-Douglas and CES, are analytic, at least on the positive orthant. ${ }^{3}$ Furthermore, as Kannai (1974) shows, the set of monotone, convex, and analytic preference orderings is dense in the set of continuous, monotone, convex preference orderings. Thus, our preference domain is a 'topologically large' subset of the standard domain of economic preferences.

For these two preference domains, we demonstrate that if the agenda domain is restricted as described above, then it is possible to satisfy all four of the choice-theoretic versions of Arrow's axioms with Weak Pareto strengthened to Strong Pareto (only strongly Pareto optimal alternatives are chosen) and Nondictatorship strengthened to Anonymity (the social choice is invariant to a permutation of the individuals' preferences). As a preliminary step in proving these theorems, we show that Independence of Infeasible Alternatives is vacuous with our domain assumptions. In order to prove this result for the case of the monotone analytic preference domain, we develop an ordinal version of the Analytic Continuation Principle for monotone analytic preferences with no critical points. The Analytic Continuation Principle says that if two analytic functions defined on an open connected set coincide on an open subset, then they must be identical.

Border (1984) has shown that no social welfare function can satisfy Arrow's axioms if the domain is the set of Euclidean spatial preference profiles when the set of alternatives is a multidimensional Euclidean space. ${ }^{4}$ However, in spatial models,

\footnotetext{
${ }^{2}$ For introductions to spatial models, see Enelow and Hinich (1984) and Ordeshook (1986).

${ }^{3}$ Our possibility theorem for monotone analytic functions is valid if the preferences are analytic on the positive orthant and can be continuously extended to the boundary of the orthant.

${ }^{4}$ Alternative proofs of Border's Theorem may be found in Le Breton and Weymark (1996) and Redekop (1993b). Redekop's version of Border's Theorem does not require the domain to include
} 
it is natural to suppose that each component of an alternative is nonnegative. Border's proof of his theorem does not apply when the set of alternatives is restricted in this way. We, therefore, establish a version of Border's impossibility theorem for the domain of all Euclidean spatial preferences when the set of alternatives is the nonnegative orthant of a multidimensional Euclidean space. We also show that for this set of alternatives, no social welfare function can satisfy Arrow's axioms if the domain is the set of profiles of strictly monotone, strictly convex, analytic preferences with no critical points. These results show the importance for our possibility theorems of being able to restrict the agenda domain. For either of the two preference domains used in our social welfare function impossibility theorems, a social choice correspondence possibility theorem is obtained with our restriction on the agenda domain.

There are relatively few articles in the Arrovian social choice literature that consider restricting the agenda domain to include only agendas that can be interpreted as being sets of feasible allocations for an economy. Of particular relevance here are the articles by Bailey (1979) and Donaldson and Weymark (1988). Bailey assumes that an agenda is the set of feasible allocations in an exchange economy. With this kind of agenda domain, Arrow's Choice Axiom is vacuous, which permits the satisfaction of all of the Arrow choice-theoretic axioms. ${ }^{5}$ Donaldson and Weymark assume that the universal set of alternatives is the nonnegative orthant in some Euclidean space and interpret alternatives as being vectors of public and/or private goods. They show that the Arrow axioms are consistent when preferences satisfy standard economic assumptions and the agenda domain contains only compact and comprehensive sets of alternatives with nonempty interiors. ${ }^{6}$ Unfortunately, the social choice correspondence Donaldson and Weymark use to establish their theorem exhibits some dictatorial features. Our possibility theorem for monotone analytic preferences shows that all vestiges of dictatorship can be avoided if preferences are also assumed to be analytic.

There has been less use of social choice correspondences in political models. Gibbard, Hylland, and Weymark (1987) show that Arrow's choice-theoretic axioms are consistent if preferences are unrestricted when every agenda is finite and contains the status quo. This agenda domain restriction is satisfied by many rules for considering motions in legislatures. Duggan (1996) supposes that the set of alternatives is a convex subset of a multidimensional Euclidean space, the agenda domain consists of all compact convex subsets of this set, and the preference domain is the set of all profiles of continuous, strictly convex preferences with an ideal point. Duggan proves that with these domain assumptions, the choice-theoretic versions of Arrow's axioms are inconsistent. Our possibility theorem for Euclidean spatial preferences therefore shows that Duggan's impossibility result depends on having non-Euclidean spatial preferences in his preference domain and on having feasible sets with no interiors in his agenda domain.

In Section 2, we introduce the social choice framework and formally define the

all profiles of Euclidean spatial preferences.

${ }^{5}$ See Donaldson and Weymark (1988) for a more detailed discussion of Bailey's contribution. Arrow's Choice Axiom is not vacuous in production economies.

${ }^{6}$ Comprehensiveness corresponds to the economic assumption of free disposal. 
axioms used in our theorems. In Sections 3 and 4, respectively, we present our impossibility and possibility theorems for Euclidean spatial preferences. In Section 5, we establish our impossibility theorem for strictly monotone, strictly convex, analytic preferences. In Section 6, we prove our ordinal version of the Analytic Continuation Principle. In Section 7, we establish our possibility theorem for monotone analytic preferences. Some concluding remarks are presented in Section 8.

\section{The framework and axioms}

The universal set of alternatives is $X$. An ordering $R$ of $X$ is a complete, reflexive, and transitive binary relation on $X$. $R$ is interpreted to be a weak preference relation. Corresponding to $R$, strict preference $P$ and indifference $I$ are defined in the usual way: (a) $x P y$ iff $x R y$ and $\neg(y R x)$ and (b) $x I y$ iff $x R y$ and $y R x$. The set of all orderings of $X$ is $\mathcal{R}$.

There is a finite set of individuals $N:=\{1, \ldots, n\}$ with $n \geq 2$. Each individual has an ordering $R_{i} \in \mathcal{R}$. A preference profile $\mathbf{R}:=\left(R_{1}, \ldots, R_{n}\right)$ is an $n$-tuple of weak preference relations, one for each person. Profiles are a priori restricted to be in $\mathcal{D} \subseteq \mathcal{R}^{n}$, the set of admissible profiles. $\mathcal{D}$ is called the preference domain. If $\mathcal{D}=\mathcal{R}^{n}$, the preference domain is unrestricted. Two profiles $\mathbf{R}^{1}, \mathbf{R}^{2} \in \mathcal{R}^{n}$ coincide on a nonempty set $A \subseteq X$ if for all $i \in N$ and all $x, y \in A, x R_{i}^{1} y$ iff $x R_{i}^{2} y$.

A social welfare function $F: \mathcal{D} \rightarrow \mathcal{R}$ is a mapping that assigns a social ordering of the alternatives in $X$ to each admissible profile. For profiles $\mathbf{R}, \mathbf{R}^{1}, \mathbf{R}^{2} \in \mathcal{D}$, we simplify the notation by letting $R, R^{1}$, and $R^{2}$ denote $F(\mathbf{R}), F\left(\mathbf{R}^{1}\right)$, and $F\left(\mathbf{R}^{2}\right)$, respectively.

Individual $d \in N$ is a dictator for the social welfare function $F$ if for all $\mathbf{R} \in \mathcal{D}$ and all $x, y \in X, x P y$ if $x P_{d} y$.

Arrow's (1963) Theorem shows that when the preference domain is unrestricted and the cardinality of $X$ is at least three, no social welfare function can satisfy the following three axioms.

Independence of Irrelevant Alternatives. For all $\mathbf{R}^{1}, \mathbf{R}^{2} \in \mathcal{D}$ and all $x, y \in X$, if $\mathbf{R}^{1}$ and $\mathbf{R}^{2}$ coincide on $\{x, y\}$, then $R^{1}$ and $R^{2}$ coincide on $\{x, y\}$.

Weak Pareto. For all $\mathbf{R} \in \mathcal{D}$ and all $x, y \in X$, if $x P_{i} y$ for all $i \in N$, then $x P y$.

Nondictatorship. No individual is a dictator.

An agenda is a nonempty subset of $X$. An agenda is a potential feasible set. The set of admissible agendas, the agenda domain, is $\mathcal{A}$.

A social choice correspondence $C: \mathcal{A} \times \mathcal{D} \rightarrow X$ is a mapping that assigns a nonempty subset of the agenda to each admissible agenda and admissible profile. That is, for all $A \in \mathcal{A}$ and all $\mathbf{R} \in \mathcal{D}, \emptyset \neq C(A, \mathbf{R}) \subseteq A$. The set $C(A, \mathbf{R})$ is called the choice set.

For a nonempty set $A \subseteq X$ and an ordering $R \in \mathcal{R}$, the set of best alternatives in $A$ according to $R$ is

$$
B(A, R):=\{x \in A \mid x R y \text { for all } y \in A\} .
$$


A social welfare function $F$ rationalizes the social choice correspondence $C$ if for all $A \in \mathcal{A}$ and all $\mathbf{R} \in \mathcal{D}, C(A, \mathbf{R})=B(A, F(\mathbf{R}))$. In other words, for each admissible agenda $A$ and admissible profile $\mathbf{R}$, the choice set consists of the best elements in $A$ according to the social preference relation $R$ associated with the profile $\mathbf{R}$.

Our independence condition for social choice correspondences requires the choice set to be independent of preferences over alternatives not in the agenda.

Independence of Infeasible Alternatives. For all $A \in \mathcal{A}$ and all $\mathbf{R}^{1}, \mathbf{R}^{2} \in \mathcal{D}$, if $\mathbf{R}^{1}$ and $\mathbf{R}^{2}$ coincide on $A$, then $C\left(A, \mathbf{R}^{1}\right)=C\left(A, \mathbf{R}^{2}\right)$.

For all $A \in \mathcal{A}$ and all $\mathbf{R} \in \mathcal{D}$, the set of strongly Pareto optimal alternatives is

$$
\begin{aligned}
\mathcal{P}(A, \mathbf{R})= & \left\{x \in A \mid \nexists y \in A \text { such that } y R_{i} x \text { for all } i \in N\right. \text { and } \\
& \left.y P_{j} x \text { for some } j \in N\right\} .
\end{aligned}
$$

The Strong Pareto axiom requires the social choice correspondence to only choose strongly Pareto optimal alternatives.

Strong Pareto. For all $A \in \mathcal{A}$ and all $\mathbf{R} \in \mathcal{D}, C(A, \mathbf{R}) \subseteq \mathcal{P}(A, \mathbf{R})$.

This axiom is a strengthening of the choice-theoretic analogue to Arrow's Weak Pareto axiom, which merely requires each choice set to be contained in the set of weakly Pareto optimal alternatives (the set of feasible alternatives for which there is no other feasible alternative that everyone strictly prefers).

Anonymity is the requirement that the social choice correspondence treats individuals symmetrically, in the following sense.

Anonymity. For all $A \in \mathcal{A}$ and all $\mathbf{R}^{1}, \mathbf{R}^{2} \in \mathcal{D}$, if $\mathbf{R}^{1}$ is a permutation of $\mathbf{R}^{2}$, then $C\left(A, \mathbf{R}^{1}\right)=C\left(A, \mathbf{R}^{2}\right)$.

This definition of Anonymity is vacuous if there are private goods and selfish preferences. We informally consider a private goods version of Anonymity in Section 7.

An individual $d \in N$ is a dictator for the social choice correspondence $C$ if $C(A, \mathbf{R}) \subseteq B\left(A, R_{d}\right)$ for all $A \in \mathcal{A}$ and all $\mathbf{R} \in \mathcal{D}$. If there are no private goods, Anonymity is more demanding than requiring there to be no dictator, provided that the preference domain is not too degenerate.

The definition of a social welfare function incorporates the social rationality condition that all social rankings of the alternatives are orderings. The analogue of this requirement for a social choice correspondence is Arrow's Choice Axiom. See Arrow (1959).

Arrow's Choice Axiom. For all $A^{1}, A^{2} \in \mathcal{A}$ and all $\mathbf{R} \in \mathcal{D}$, if $A^{1} \subset A^{2}$ and $C\left(A^{2}, \mathbf{R}\right) \cap$ $A^{1} \neq \emptyset$, then $C\left(A^{1}, \mathbf{R}\right)=C\left(A^{2}, \mathbf{R}\right) \cap A^{1}$.

Hansson (1968) has shown that if $\mathcal{A}$ is closed under finite unions, then Arrow's Choice Axiom is equivalent to requiring the social choice correspondence to be rationalizable by a social welfare function. 
In its choice-theoretic formulation, Arrow's Theorem assumes that the preference domain is unrestricted and the agenda domain includes all of the subsets of alternatives that contain two or three alternatives. Provided that there are at least three alternatives, this version of Arrow's Theorem shows that there is no social choice correspondence that satisfies Independence of Infeasible Alternatives, Arrow's Choice Axiom, and the choice-theoretic versions of the Weak Pareto and Nondictatorship axioms. ${ }^{7}$ A fortiori, there is no social choice correspondence that satisfies Independence of Infeasible Alternatives, Arrow's Choice Axiom, Strong Pareto, and Anonymity either.

\section{An impossibility theorem for Euclidean spatial preferences}

Border's (1984) social welfare function impossibility theorem for Euclidean spatial preferences supposes that the set of alternatives $X$ is $\mathbb{R}^{m}$ with $m \geq 2$. However, in spatial models, it is natural to suppose that that the set of alternatives is $\mathbb{R}_{+}^{m}$, not all of $\mathbb{R}^{m}{ }^{8}$ In this section, we establish a version of Border's impossibility theorem when the set of alternatives is restricted in this way.

A preference ordering $R$ on $X \subseteq \mathbb{R}^{m}$ is a Euclidean spatial preference if for some $b \in X, x R y$ iff $\|x-b\| \leq\|y-b\|$, where $\|\cdot\|$ denotes the Euclidean norm. The indifference surfaces of a Euclidean spatial preference are the intersections with $X$ of spheres (circles in two dimensions) centered at an ideal or bliss point $b$. Note that we have required the bliss point to be in $X$. A profile $\mathbf{R} \in \mathcal{R}^{n}$ is a Euclidean spatial profile if $R_{i}$ is a Euclidean spatial preference for all $i \in N$. Let $\mathcal{S}$ be the set of all Euclidean spatial preferences.

We assume that the set of alternatives is $X=\mathbb{R}_{+}^{m}$ with $m \geq 2$. $X$ can be interpreted as being an issue space with each component indicating the quantity or magnitude of some issue. For example, one component might be the government's expenditure on education. In spatial models of elections, a voter has a spatial preference on this issue space and votes for the candidate whose platform (a point in the issue space) is closest to his or her ideal point. In spatial models of legislatures, the preferences of the legislators (which in turn might depend on voters' preferences) are used to choose between alternatives in the issue space.

Border's Theorem shows that when $X=\mathbb{R}^{m}$ and $m \geq 2$, no social welfare function with preference domain $\mathcal{S}^{n}$ can satisfy Arrow's three axioms. Our impossibility theorem shows that the same conclusion holds when the set of alternatives is $\mathbb{R}_{+}^{m}$.

Theorem 1. If $X=\mathbb{R}_{+}^{m}$ with $m \geq 2$, there does not exist a social welfare function with preference domain $\mathcal{S}^{n}$ that satisfies Independence of Irrelevant Alternatives, Weak Pareto, and Nondictatorship.

Our proof of Theorem 1 makes use of a theorem about saturating preference domains due to Kalai, Muller, and Satterthwaite (1979). The preference domain $\mathcal{D}$

\footnotetext{
${ }^{7}$ See Donaldson and Weymark (1988) for a formal statment of this theorem.

${ }^{8} \mathbb{R}_{+}^{m}$ and $\mathbb{R}_{++}^{m}$ are the nonnegative and positive orthants, respectively, of the $m$-dimensional Euclidean space $\mathbb{R}^{m}$.
} 
is common if there exists a set of preferences $\mathcal{E} \subseteq \mathcal{R}$ such that $\mathcal{D}=\mathcal{E}^{n}$. Suppose that $\mathcal{D}$ is a common preference domain with individual preference domain $\mathcal{E}$. For a set $A \subseteq X$, let $\left.\mathcal{E}\right|_{A}$ and $\left.\mathcal{R}\right|_{A}$ be the restrictions of $\mathcal{E}$ and $\mathcal{R}$, respectively, to $A$. A pair of alternatives $x, y \in X$ is a trivial pair if the cardinality of $\left.\mathcal{E}\right|_{\{x, y\}}$ is one; i.e., in $\mathcal{E}$, there is only one way $x$ and $y$ are ranked. A pair of alternatives $x, y \in X$ is nontrivial if it is not a trivial pair. The set $\{x, y, z\} \subseteq X$ is a free triple if $\left.\mathcal{E}\right|_{\{x, y, z\}}=\left.\mathcal{R}\right|_{\{x, y, z\}}$; i.e., if the individual preference domain is unrestricted on $\{x, y, z\}$. Two pairs of alternatives $A$ and $B$ contained in $X$ are connected if there exists a finite sequence $C_{1}, \ldots, C_{r}$ of pairs contained in $X$ such that $C_{1}=A, C_{r}=B$, and $C_{i} \cup C_{i+1}$ is a free triple for all $i=1, \ldots, r-1$. A common preference domain $\mathcal{D}$ is saturating if (i) there exists at least two nontrivial pairs and (ii) any two nontrivial pairs are connected. The Kalai-Muller-Satterthwaite Theorem says that no social welfare function defined on a common saturating preference domain can satisfy Independence of Irrelevant Alternatives, Weak Pareto, and Nondictatorship. ${ }^{9}$

With the individual preference domain $\mathcal{S}$, every pair of alternatives is nontrivial. Hence, to establish Theorem 1, it is sufficient to show that any two pairs of alternatives can be connected to each other. ${ }^{10}$

Proof of Theorem 1. The proof proceeds in a series of steps. The various constructions used in the proof are illustrated in Figure 1.

Step 1. We first show that for any distinct $x, y \in \mathbb{R}_{+}^{m}$, there exists a $z \in \mathbb{R}_{++}^{m}$ such that $\|x-z\|=\|y-z\|$. If $(x+y) / 2 \in \mathbb{R}_{++}^{m}$, simply set $z=(x+y) / 2$. Otherwise, let $L(x, y)$ be the line segment joining $x$ and $y$ and $H(x, y)$ be the $(m-1)$-dimensional hyperplane orthogonal to $L(x, y)$ through $(x+y) / 2$. If $H(x, y)$ does not intersect $\mathbb{R}_{++}^{m}$, then for some $i, w_{i}=0$ for all $w \in H(x, y)$. Because $(x+y) / 2 \in H(x, y)$, $L(x, y)$ is othogonal to $H(x, y)$, and neither $x$ nor $y$ is in $H(x, y)$, it follows that $x_{i}=-y_{i} \neq 0$, contradicting the assumption that $x$ and $y$ are both in $\mathbb{R}_{+}^{m}$. Hence, $H(x, y)$ intersects $\mathbb{R}_{++}^{m}$. By letting $z$ be any point in this intersection, we complete the proof of Step 1.

Step 2. Suppose that $w, x$, and $y$ are distinct points in $\mathbb{R}_{+}^{m}$. It follows from Lemma 2 in Campbell (1993) that if there exists a $z \in \mathbb{R}_{++}^{m}$ such that $\|w-z\|=$ $\|x-z\|=\|y-z\|$, then $\{w, x, y\}$ is a free triple.

Step 3. Consider any distinct $x^{1}, x^{2} \in \mathbb{R}_{+}^{m}$. By Step 1 , there exists an $x \in \mathbb{R}_{++}^{m}$ and an $\varepsilon_{x}>0$ such that $x^{1}$ and $x^{2}$ lie on a sphere of radius $\varepsilon_{x}$ centered at $x$. Let $C_{x}$ be the restriction to $\mathbb{R}_{+}^{m}$ of this sphere. The ray from the origin through $x$ intersects $C_{x}$ either once or twice. In the first case, let $a$ be this point of intersection and, in the second case, let $a$ be the point of intersection farthest from the origin.

Next, consider any distinct $y^{1}, y^{2} \in \mathbb{R}_{+}^{m}$. By the preceding argument, $y^{1}$ and $y^{2}$ lie on a sphere centered at $y$. Let $C_{y}$ be the restriction to $\mathbb{R}_{+}^{m}$ of this sphere and $b$ be the point farthest from the origin in $C_{y}$ on the ray through $y$. In this and the following two steps, we consider the case in which $x$ and $y$ are not proportional to each other.

\footnotetext{
${ }^{9}$ In this theorem, $X$ can be any set.

${ }^{10}$ Le Breton and Weymark (1996) use the Kalai-Muller-Satterthwaite Theorem to prove the original version of Border's Theorem, but their proof does not apply when the alternatives are restricted to being in $\mathbb{R}_{+}^{m}$.
} 
We now construct a sphere $A_{1}$ centered at $a$. There is a critical value $\varepsilon_{a}>0$ such that any sphere of radius less than $\varepsilon_{a}$ centered at $a$ is entirely contained in $\mathbb{R}_{++}^{m}$. By choosing the radius of $A_{1}$ to be less than $\operatorname{Min}\left\{\varepsilon_{x}, \varepsilon_{a}\right\}$, we have $\left|C_{x} \cap A_{1}\right| \geq 2$. Further, we can choose the radius of $A_{1}$ so that it is less than $\|(a-b) / 2\|$ and so that $C_{x} \cap A_{1} \cap\left(x^{1} \cup x^{2}\right)=\emptyset$.

Similarly, we can construct a sphere $A_{2}$ centered at $b$ with the following three properties: (i) the radius of $A_{2}$ is less than $\|(a-b) / 2\|$, (ii) $\left|C_{y} \cap A_{2}\right| \geq 2$, and (iii) $C_{y} \cap A_{2} \cap\left(y^{1} \cup y^{2}\right)=\emptyset$.

Let $s^{1}$ and $s^{2}$ be distinct points in $C_{x} \cap A_{1}$ and $s^{7}$ and $s^{8}$ be distinct points in $C_{y} \cap A_{2}$.

Step 4. Let $L(a, b)$ be the line segment joining $a$ and $b$. Let $\bar{a}$ be the point of intersection of $A_{1}$ and $L(a, b)$ and $\bar{b}$ be the point of intersection of $A_{2}$ and $L(a, b)$. Because the radii of $A_{1}$ and $A_{2}$ are both less than $\|(a-b) / 2\|$, a sphere centered at $c=(\bar{a}+\bar{b}) / 2$ with radius $\|(\bar{a}-\bar{b}) / 2\|$ is tangent to $A_{1}$ at $\bar{a}$ and tangent to $A_{2}$ at $\bar{b}$. By increasing this radius slightly, we can construct a sphere $A_{3}$ centered at $c$ with the following four properties: (i) $\left|A_{1} \cap A_{3}\right| \geq 2$, (ii) $A_{1} \cap A_{3} \cap\left(s^{1} \cup s^{2}\right)=\emptyset$, (iii) $\left|A_{2} \cap A_{3}\right| \geq 2$, and (iv) $A_{2} \cap A_{3} \cap\left(s^{7} \cup s^{8}\right)=\emptyset$.

Let $s^{3}$ and $s^{4}$ be distinct points in $A_{1} \cap A_{3}$ and $s^{5}$ and $s^{6}$ be distinct points in $A_{2} \cap A_{3}$.

Step 5. Because $x^{1}, x^{2}, s^{1}, s^{2} \in C_{x}$, by Step 2, $\left\{x^{1}, x^{2}, s^{1}\right\}$ and $\left\{x^{2}, s^{1}, s^{2}\right\}$ are free triples. Similarly, because $s^{1}, s^{2}, s^{3}, s^{4} \in A_{1},\left\{s^{1}, s^{2}, s^{3}\right\}$ and $\left\{s^{2}, s^{3}, s^{4}\right\}$ are free triples. Because $s^{3}, s^{4}, s^{5}, s^{6} \in A_{3},\left\{s^{3}, s^{4}, s^{5}\right\}$ and $\left\{s^{4}, s^{5}, s^{6}\right\}$ are free triples. Because $s^{5}, s^{6}, s^{7}, s^{8} \in A_{2},\left\{s^{5}, s^{6}, s^{7}\right\}$ and $\left\{s^{6}, s^{7}, s^{8}\right\}$ are free triples. Because $s^{7}, s^{8}, y^{1}, y^{2} \in$ $C_{y},\left\{s^{7}, s^{8}, y^{1}\right\}$ and $\left\{s^{8}, y^{1}, y^{2}\right\}$ are free triples. We have thus shown that the pair $\left\{x^{1}, x^{2}\right\}$ is connected to the pair $\left\{y^{1}, y^{2}\right\}$ when $x$ and $y$ are not proportional to each other.

Step 6. Now suppose that $x$ and $y$ are proportional. Consider any point $w \in \mathbb{R}_{++}^{m}$ that is not proportional to $x$ and $y$. Let $w^{1}$ and $w^{2}$ be distinct points in $\mathbb{R}_{+}^{m}$ equidistant from $w$. By Step 5, $\left\{x^{1}, x^{2}\right\}$ is connected to $\left\{w^{1}, w^{2}\right\}$ and $\left\{w^{1}, w^{2}\right\}$ is connected to $\left\{y^{1}, y^{2}\right\}$. Hence, $\left\{x^{1}, x^{2}\right\}$ is connected to $\left\{y^{1}, y^{2}\right\}$ even if $x$ and $y$ are proportional to each other.

Step 7. We have shown that any pair of alternatives is connected to any other pair of alternatives. Hence, the domain is saturating, and so by the Kalai-MullerSatterthwaite (1979) Theorem, Arrow's three axioms are inconsistent.

\section{A possibility theorem for Euclidean spatial preferences}

In this section, we present a social choice correspondence possibility theorem for Euclidean spatial preferences. We escape from Arrow's choice-theoretic impossibility by restricting our preference domain to include only Euclidean spatial preferences and by restricting our agenda domain to contain only compact sets with nonempty interiors. As in the previous section, we suppose that $X=\mathbb{R}_{+}^{m}$ with $m \geq 2$.

Our possibility theorem holds for any domain of Euclidean spatial preferences, not just the set of all Euclidean spatial profiles. We do not require the preference domain to be common. 
Euclidean Spatial Preference Domain. $\mathcal{D} \subseteq \mathcal{S}^{n}$.

We also restrict the set of potential agendas. We require each agenda to have an interior, and thus rule out the possibility of an agenda consisting solely of a discrete set of points. We also require each agenda to be compact. These assumptions are satisfied, for example, in the legislative interpretation of the spatial model if the agenda is determined by a limited legislative budget. Note that we do not require agendas to be connected.

Full-Dimensional Compact Agenda Domain. For all $A \in \mathcal{A}, A$ is compact and has a nonempty interior.

Because our possibility theorem holds for any Euclidean spatial preference domain and any full-dimensional compact agenda domain, it is possible to place further restrictions on these domains. For example, we could also assume that all agendas are comprehensive and convex.

Lemma 1 demonstrates that Independence of Infeasible Alternatives is vacuous in the presence of our two domain restrictions. This lemma plays a key role in our possibility theorem.

Lemma 1. If $X=\mathbb{R}_{+}^{m}$ with $m \geq 2$ and the social choice correspondence $C$ is defined on a Euclidean spatial preference domain and a full-dimensional compact agenda domain, then $C$ satisfies Independence of Infeasible Alternatives. ${ }^{11}$

Proof. First, note that with a Euclidean spatial preference ordering $R$, it is possible to determine the ideal point $b$ from knowledge of any segment of an indifference surface when $m \geq 2 .{ }^{12}$ Simply take two points on the indifference surface and see where the lines perpendicular to the indifference surface at these points intersect. In other words, the complete preference ordering $R$ can be uniquely determined from a segment of an indifference surface. Thus, if $R^{1}$ and $R^{2}$ coincide on $A \in \mathcal{A}$, because $A$ has a nonempty interior, $R^{1}$ and $R^{2}$ must coincide on all of $X$. Hence, Independence of Infeasible Alternatives is trivially satisfied.

Theorem 2 demonstrates that our four axioms for social choice correspondences are consistent on a Euclidean spatial preference domain when there is a full-dimensional compact agenda domain and the set of alternatives is at least two dimensional.

Theorem 2. If $X=\mathbb{R}_{+}^{m}$ with $m \geq 2$, on any Euclidean spatial preference domain and any full-dimensional compact agenda domain, there exist social choice correspondences that satisfy Independence of Infeasible Alternatives, Strong Pareto, Anonymity, and Arrow's Choice Axiom.

\footnotetext{
${ }^{11}$ This lemma is also valid without the assumption that agendas are compact.

${ }^{12}$ In one dimension, if the preference ordering is only known for a segment on one side of the ideal point, it is not possible to determine the location of the ideal point. A one-dimensional Euclidean spatial preference ordering is a special kind of single-peaked preference.
} 
Proof. For each Euclidean spatial preference $R$ in $\mathcal{S}$, choose a continuous utility function $U^{R}$ to represent $R .^{13}$ This representation is fixed throughout the proof. Let $W: \mathbb{R}^{n} \rightarrow \mathbb{R}$ be any continuous symmetric function, increasing in each of its arguments. Using $W$, we construct a social welfare function $F$ by setting, for all $\mathbf{R} \in \mathcal{D}$ and all $x, y \in X$,

$$
x F(\mathbf{R}) y \quad \text { iff } W\left[U^{R_{1}}(x), \ldots, U^{R_{n}}(x)\right] \geq W\left[U^{R_{1}}(y), \ldots, U^{R_{n}}(y)\right] .
$$

Because $W$ and $U^{R_{i}}, i \in N$, are continuous functions, $F(\mathbf{R})$ is a continuous ordering. We use $F$ to define a social choice correspondence $C: \mathcal{A} \times \mathcal{D} \rightarrow X$ that satisfies all of the axioms of the theorem. For all $(A, \mathbf{R}) \in \mathcal{A} \times \mathcal{D}$, let $C(A, \mathbf{R})=B(A, F(\mathbf{R}))$. In words, $C(A, \mathbf{R})$ is the set of best alternatives in $A$ according to the social preference ordering corresponding to the profile $\mathbf{R}$. Because $A$ is compact and $F(\mathbf{R})$ is a continuous ordering, $C$ is well-defined for all $(A, \mathbf{R}) \in \mathcal{A} \times \mathcal{D}$. By Lemma $1, C$ satisfies Independence of Infeasible Alternatives. Because $W$ is increasing in its arguments, $C$ satisfies Strong Pareto. Because for each Euclidean spatial preference $R$ there is a fixed utility representation $U^{R}$ and because $W$ is symmetric in its arguments, $C$ satisfies Anonymity. Because $C$ is rationalized by the social welfare function $F$, it satisfies Arrow's Choice Axiom.

A Bergson-Samuelson social welfare function is a real-valued function defined on $n$-tuples of utilities. The function $W$ used in the proof of Theorem 2 is a BergsonSamuelson social welfare function. Thus, we are using a welfare function of the kind found in traditional welfare economics to generate our social choice correspondence.

Welfarism requires the social desirability of an alternative to be determined solely on the basis of the individual utilities obtained with it. ${ }^{14}$ Because the social choice correspondence $C$ used to establish Theorem 2 is constructed from a Bergson-Samuelson social welfare function, it is welfarist. Example 1 shows how to construct a nonwelfarist social choice correspondence satisfying the assumptions of Theorem 2.

Example 1. ${ }^{15}$ Let $C$ be a social choice correspondence constructed as in the proof of Theorem 2. We simply assume that $C$ is not a function, rather than placing restrictions on the domains that ensure that this is the case. A new social choice correspondence $C^{1}: \mathcal{A} \times \mathcal{D} \rightarrow X$ is constructed by selecting the alternatives with the largest first components in each choice set of $C$. Formally, for all $A \in \mathcal{A}$ and all $\mathbf{R} \in \mathcal{D}, C^{1}(A, \mathbf{R})=\left\{x \in C(A, \mathbf{R}) \mid x_{1} \geq y_{1}\right.$ for all $\left.y \in C(A, \mathbf{R})\right\}$. Because $C$ is generated by a social welfare function with continuous social preferences and because each agenda is compact, $C(A, \mathbf{R})$ is compact. Hence, $C^{1}$ is well-defined. $C^{1}$ clearly satisfies Independence of Infeasible Alternatives (by Lemma 1), Strong Pareto (because it is a subcorrespondence of $C$ ), and Anonymity (because the tie-breaking rule is anonymous). For a fixed profile $\mathbf{R}, C^{1}$ is rationalized by a lexicographic

\footnotetext{
${ }^{13}$ A function $U: X \rightarrow \mathbb{R}$ is a utility function representing the preference ordering $R \in \mathcal{R}$ if for all $x, y \in X, U(x) \geq U(y)$ iff $x R y$.

${ }^{14}$ For discussions of welfarism, see Bossert and Weymark (1996), d'Aspremont (1985), and Sen (1979).

${ }^{15}$ We are indebted to Yves Sprumont for this example.
} 
ordering - alternatives are first ranked according to $F(\mathbf{R})$ and then by the magnitudes of their first components. Thus, $C^{1}$ also satisfies Arrow's Choice Axiom.

Our possibility theorem for social choice correspondences is in sharp contrast to our spatial impossibility theorem for social welfare functions. The social welfare function $F$ used in the proof of Theorem 2 rationalizes the social choice correpondence $C$ we constructed. It follows from Theorem 1 that if the preference domain is $\mathcal{S}^{n}$, $F$ cannot satisfy all three of Arrow's axioms. F satisfies Nondictatorship (because it treats individuals symmetrically) and Weak Pareto, so it does not satisfy Independence of Irrelevant Alternatives. If all the binary agendas were in the agenda domain, it would not be possible to rationalize a social choice correspondence satisfying Independence of Infeasible Alternatives by a social welfare function unless the social welfare function satisfies Independence of Irrelevant Alternatives.

\section{An impossibility theorem for monotone analytic preferences}

Classical economic preferences are continuous, monotone, and convex. Redekop (1993a) has shown that in order for a social welfare function defined on a domain of profiles of classical economic preferences to satisfy Arrow's axioms when the set of alternatives is the nonnegative orthant of a multidimensional Euclidean space, the domain must be a topologically negligible subset of the classical economic domain. In this section, we use Redekop's results to show that Arrow's axioms are inconsistent on the domain of profiles of strictly monotone, strictly convex, analytic preferences with no critical points. As in our spatial model, we assume that $X=\mathbb{R}_{+}^{m}$ with $m \geq 2$. Alternatives in $X$ can be interpreted as being vectors of public goods.

A preference ordering $R$ on a subset $K$ of $\mathbb{R}_{+}^{m}$ is monotone if $x P y$ for all $x, y \in K$ such that $x \gg y$ and it is strictly monotone if $x P y$ for all distinct $x, y \in K$ such that $x \geq y$. A real-valued function $f$ defined on a subset $K$ of $\mathbb{R}^{m}$ is monotone (strictly monotone) if $f(x)>f(y)$ for all distinct $x, y \in K$ such that $x \gg y(x \geq y)$. Any utility function representing a (strictly) monotone preference ordering is (strictly) monotone.

A preference ordering $R$ on a convex subset $K$ of $\mathbb{R}_{+}^{m}$ is convex if for all $x \in K$, $\{y \in K \mid y R x\}$ is a convex set and it is strictly convex if for all $x \in K,\{y \in K \mid y R x\}$ is a strictly convex set relative to $K$.

A real-valued function $f$ defined on an open subset $K$ of $\mathbb{R}^{m}$ is analytic if for each $x \in K$, there exists a Taylor's series in powers of $y-x$ whose sum is equal to the value of $f$ at each point $y$ in some neighbourhood of $x .^{16}$ A real-valued function $f$ defined on a subset $K$ of $\mathbb{R}^{m}$ is analytic if for all $x \in K$, there exists an open subset $V$ of $\mathbb{R}^{m}$ and an analytic function $g: V \rightarrow \mathbb{R}$ such that $\left.g\right|_{K \cap V}=\left.f\right|_{K \cap V}$. Analytic functions are infinitely continuously differentiable. A preference ordering $R$ on a set $K$ in $\mathbb{R}_{+}^{m}$ is analytic if it can be represented by an analytic utility function.

A differentiable real-valued function $f$ on a convex set $K$ in $\mathbb{R}^{m}$ has a critical point at $a \in K$ if the gradient of $f$ vanishes at $a$. A preference ordering $R$ on a convex

\footnotetext{
${ }^{16}$ For a more formal definition of an analytic function on an open subset of $\mathbb{R}^{m}$, see Dieudonné (1969, p. 203).
} 
set $K$ in $\mathbb{R}_{+}^{m}$ has no critical points if there exists a differentiable utility function $f$ representing $R$ that has no critical points.

Let $\mathcal{M}_{c}$ denote the set of all strictly monotone, strictly convex, analytic preferences with no critical points. We now show that with the preference domain $\mathcal{M}_{c}^{n}$, no social welfare function can satisfy Arrow's three axioms.

Theorem 3. If $X=\mathbb{R}_{+}^{\mathrm{m}}$ with $m \geq 2$, there does not exist a social welfare function with preference domain $\mathcal{M}_{c}^{n}$ that satisfies Independence of Irrelevant Alternatives, Weak Pareto, and Nondictatorship.

Proof. Let $\mathcal{D}=\mathcal{E}^{n}$ be a common preference domain and $\mathcal{P}$ be the set of all continuous, strictly monotone, strictly convex preferences on $X$. $\mathcal{E}$ is a near-open subset of $\mathcal{P}$ if there exists an open set of preferences $\mathcal{O} \subseteq \mathcal{P}$ such that $\overline{\mathcal{E}}=\overline{\mathcal{O}}$. In this definition, open and closed sets are defined using the Kannai (1970) topology on $\mathcal{P} .{ }^{17}$ In particular, $\mathcal{E}$ is a near-open subset of $\mathcal{P}$ if $\mathcal{E}$ is a dense subset of $\mathcal{P}$. ${ }^{18}$ Redekop (1993a, Theorem 3.1) shows that Independence of Irrelevant Alternatives, Weak Pareto, and Nondictatorship are inconsistent if $\mathcal{E}$ is a near-open subset of $\mathcal{P}$.

Kannai (1974) has shown that the set of monotone, convex, analytic preferences on $\mathbb{R}_{+}^{m}$ is dense in the set of continuous, monotone, convex preference orderings on $\mathbb{R}_{+}^{m}$. He does this by showing that for any continuous, monotone, convex preference ordering on $\mathbb{R}_{+}^{m}$, there exists a sequence of analytic, monotone, concave utility functions that converge to a utility function representing the given preference ordering. We adapt Kannai's argument to show that $\mathcal{M}_{c}$ is dense in $\mathcal{P}$. Let $R$ be an arbitrary preference in $\mathcal{P}$. Applying Kannai's argument, there exists a sequence $\left\langle U^{k}\right\rangle$ of analytic, monotone, concave utility functions that converge to a utility function $U$ representing $R$. Let $V: \mathbb{R}_{+}^{m} \rightarrow \mathbb{R}$ be a strictly monotone, strictly concave, analytic function with no critical points. Then, for all $k, V^{k}=U^{k}+\frac{1}{k} V$ is a strictly monotone, strictly concave, analytic function with no critical points. The limit of the sequence $\left\langle V^{k}\right\rangle$ is $U$. For all $k, V^{k}$ represents a preference in $\mathcal{M}_{c}$. Hence, we have shown that $\mathcal{M}_{c}$ is dense in $\mathcal{P}$. It then follows from Redekop's Theorem that Arrow's axioms are inconsistent on the preference domain $\mathcal{M}_{c}^{n}$.

In a private goods economy, an individual is selfish if he or she only cares about his or her own consumption. Because the domain of individual preferences $\mathcal{M}_{c}$ only includes strictly monotone preferences, Theorem 3 does not apply when there are private goods and individuals are selfish. Suppose $m=\bar{m} n$ where $\bar{m}$ is the number of private goods and $X=Y^{n}$ where $Y \subseteq \mathbb{R}^{\bar{m}}$. An allocation is then a vector $\left(x^{1}, \ldots, x^{n}\right)$ where $x^{i} \in Y$ is person $i$ 's private consumption vector. By adapting an example in Blau (1957), Border (1983) has shown that Arrow's three axioms are consistent if $Y=\mathbb{R}_{+}^{\bar{m}}$ and the preference domain consists of all profiles of selfish preferences that are continuous and strictly monotone when restricted to $Y$. With the further

\footnotetext{
${ }^{17}$ See Redekop (1993c) for a definition of the Kannai topology. Redekop shows that on the domain of all continuous monotone preferences, the Kannai topology coincides with the topology of closed convergence and with Redekop's questionnaire topology.

${ }^{18} \mathrm{~A}$ set $A$ is dense in $B$ if $B \subseteq \bar{A}$.
} 
assumption that preferences are convex, Border (1984) and Maskin (1976) have shown that Arrow's axioms are inconsistent on the subset of alternatives $\mathbb{R}_{++}^{\bar{m} n}$ when $\bar{m} \geq 2$. In other words, on this preference domain, any social welfare function satisfying Independence of Irrelevant Alternatives and Weak Pareto must be dictatorial on the set of all allocations in which everyone has a positive consumption of each good. ${ }^{19}$ Redekop (1993a) has shown that these results also hold for any domain of selfish preferences in which, restricted to $Y$, the domain of individual preferences is near-open as a subset of the set of all continuous, strictly monotone, strictly convex preferences on $Y$. Using Redekop's theorems, it is straightforward to adapt the proof of Theorem 3 to show that these private goods possibility and impossibility theorems are also valid for selfish individuals whose individual preference domain restricted to $Y$ is $\mathcal{M}_{c}$.

\section{An Ordinal Analytic Continuation Principle}

To help establish our possibility theorem for monotone analytic preferences, we need to develop an ordinal version of the Analytic Continuation Principle. A statement of the standard Analytic Continuation Principle is given in Theorem 4.

Theorem 4. [Dieudonné (1969, p. 208; 1971, p. 157)] Let $f^{1}$ and $f^{2}$ be two realvalued analytic functions defined on an open connected subset $X$ of $\mathbb{R}^{\mathrm{m}}$. If there is a nonempty open subset $K$ of $X$ such that $f^{1}(x)=f^{2}(x)$ for all $x \in K$, then $f^{1}(x)=f^{2}(x)$ for every $x \in X$.

Informally, this principle says that if two analytic functions defined on an open connected set coincide on an open subset, then they must be identical. In other words, there is a unique analytic extension of an analytic function defined on an open set to an open connected superset.

Two real-valued functions $f^{1}$ and $f^{2}$ defined on a set $S \subseteq \mathbb{R}^{m}$ are ordinally equivalent if there exists an increasing real-valued function $g$ defined on $f^{1}(S)$ such that $f^{2}(x)=g\left(f^{1}(x)\right)$ for every $x \in S$. A natural question is: If $f^{1}$ and $f^{2}$ are two analytic functions defined on an open connected set $X$ and if $f^{1}$ and $f^{2}$ are ordinally equivalent on an open subset $K$ of $X$, must $f^{1}$ and $f^{2}$ also be ordinally equivalent on $X$ ? Unfortunately, the answer to this question is no, as the following simple example demonstrates.

Example 2. Let $X=\mathbb{R}$ and $K=(0,1)$. The functions $f^{1}$ and $f^{2}$ are defined by setting $f^{1}(x)=x$ and $f^{2}(x)=x^{2}$ for all $x \in \mathbb{R}$. Because both of these functions are polynomials, they are analytic. Both $f^{1}$ and $f^{2}$ are increasing on $K$, and so are ordinally equivalent on this set. However, they are not ordinally equivalent on $X$.

By requiring the functions in Theorem 4 to be monotone with no critical points, we obtain an ordinal version of the Analytical Continuation Principle when $X=\mathbb{R}_{++}^{m}$. This result is stated as Theorem 5 .

\footnotetext{
${ }^{19}$ In fact, Arrow's axioms are inconsistent on the set of alternatives in which everyone consumes a positive amount of at least one good. See Le Breton and Weymark $(1996,2001)$ for detailed discussions of the consistency of Arrow's axioms in private goods economies.
} 
Theorem 5. Let $f^{1}$ and $f^{2}$ be two real-valued, monotone, analytic functions with no critical points defined on $\mathbb{R}_{++}^{\mathrm{m}}$. If there is a nonempty open subset $K$ of $\mathbb{R}_{++}^{\mathrm{m}}$ such that $f^{1}$ and $f^{2}$ are ordinally equivalent on $K$, then $f^{1}$ and $f^{2}$ are ordinally equivalent on $\mathbb{R}_{++}^{\mathrm{m}}$.

Proof. For all $x \in \mathbb{R}_{++}^{\mathrm{m}}$, let

$$
g^{1}(x)=\frac{\nabla f^{1}(x)}{\left\|\nabla f^{1}(x)\right\|}
$$

and

$$
g^{2}(x)=\frac{\nabla f^{2}(x)}{\left\|\nabla f^{2}(x)\right\|}
$$

Because $f^{1}$ and $f^{2}$ have nonvanishing gradients, the vector-valued functions $g^{1}$ and $g^{2}$ are well-defined. The functions $g^{1}$ and $g^{2}$ are the normalized gradient functions for the functions $f^{1}$ and $f^{2}$. Analycity is preserved by partial differentiation and by the operations used to normalize the gradient functions [see Dieudonné (1969, pp. 203-205; 1971, pp. 154-159)], so the functions $g^{1}$ and $g^{2}$ are analytic (in each of their components) on $\mathbb{R}_{++}^{\mathrm{m}}$. Because $f^{1}$ and $f^{2}$ are ordinally equivalent on $K$, it follows that $g^{1}$ and $g^{2}$ coincide on $K$. Applying Theorem 4 component by component to $g^{1}$ and $g^{2}$, we can then conclude that $g^{1}(x)=g^{2}(x)$ for all $x \in \mathbb{R}_{++}^{\mathrm{m}}$. It then follows from Debreu's $\left(1972\right.$, p. 606) theorem on monotone $C^{\infty}$ functions, as corrected in Debreu (1976), that $f^{1}$ is ordinally equivalent to $f^{2}$ on $X .^{20}$

Theorem 5 can be interpreted as being a theorem about analytic, monotone, ordinal utility functions with no critical points defined on the set of consumption bundles with positive components. In this interpretation, the theorem says that if one knows the functional form of a utility function on an open subset of the positive orthant, then there is a unique (up to a monotone transform) extension of the utility function to all of $\mathbb{R}_{++}^{m}$.

When $m=1$, Theorem 5 can be strengthened by only requiring $f^{1}$ and $f^{2}$ to be monotone increasing or monotone decreasing; it is not necessary to assume that they are analytic or even differentiable. Obviously, if a function is known to be monotone increasing or monotone decreasing, which of these cases prevails can be determined from knowledge of the function on any open interval. However, because the class of admissible functions is so restricted in the one-dimensional case, this one-dimensional result is fairly trivial.

\section{A possibility theorem for monotone analytic preferences}

In this section, we use the ordinal version of the Analytic Continuation Principle presented in the previous section to develop a social choice correspondence possibility theorem for monotone analytic preferences with no critical points. As in our

\footnotetext{
${ }^{20}$ Debreu's theorem essentially says that if one has a monotone $C^{\infty}$ function with no critical point defined on the positive orthant, then the function is completely characterized, up to a monotonic transform, by its normalized gradient function. To apply Debreu's result, $g^{1}$ and $g^{2}$ must satisfy the usual integrability conditions [(2) in Debreu (1972)]. Because we have derived the normalized gradient functions $g^{1}$ and $g^{2}$ from the functions $f^{1}$ and $f^{2}$, this condition is satisfied.
} 
impossibility theorem for monotone analytic preferences, we suppose that the set of alternatives is $\mathbb{R}_{+}^{m}$.

Let $\mathcal{M}$ denote the set of all monotone analytic preferences with no critical points. We assume that the preference domain is a subset of $\mathcal{M}^{n}$.

Monotone Analytic Preference Domain. $\mathcal{D} \subseteq \mathcal{M}^{n}$.

We do not require $\mathcal{D}$ to consist of all profiles of monotone analytic preferences with no critical points. Consequently, it is possible to require that the admissible profiles satisfy further regularity conditions in addition to monotonicity and analycity. For example, the preference domain could be the domain $\mathcal{M}_{c}^{n}$ used in Theorem 3 , in which case all preferences are strictly monotone and strictly convex. We do not require the preference domain to be common.

As in our possibility theorem for Euclidean spatial preferences, we assume that the social choice correspondence has a full-dimensional compact agenda domain. With monotone preferences, in general, Pareto optimality and nonemptiness of the choice set are incompatible if the agenda is not compact. Further restrictions can be placed on the agenda domain. For example, agendas could be restricted to be also comprehensive and convex. Such agendas can be interpreted as being the set of feasible allocations of public goods obtainable with convex production technologies from a given endowment of initial resources.

In Section 4, we showed that Independence of Infeasible Alternatives is vacuous for a Euclidean spatial preference domain and a full-dimensional compact agenda domain. The same result holds for a monotone analytic preference domain and a full-dimensional compact agenda domain.

Lemma 2. If $X=\mathbb{R}_{+}^{\mathrm{m}}$ with $m \geq 2$ and the social choice correspondence $C$ is defined on a monotone analytic preference domain and a full-dimensional compact agenda domain, then $C$ satisfies Independence of Infeasible Alternatives.

Proof. Suppose that $\mathbf{R}^{1}$ and $\mathbf{R}^{2}$ coincide on $A \in \mathcal{A}$. Because these profiles are monotone and analytic with no critical points, for all $i \in N$ and for $j=1,2, R_{i}^{j}$ can be represented by a monotone analytic utility function with no critical point, $U_{i}^{j}$. Because $A$ has a nonempty interior, there exists an open subset $K$ of $A$. Because $\mathbf{R}^{1}$ and $\mathbf{R}^{2}$ coincide on $A$, for all $i \in N, U_{i}^{1}$ is ordinally equivalent to $U_{i}^{2}$ on $K$. Thus, by Theorem 5, for all $i \in N, U_{i}^{1}$ is ordinally equivalent to $U_{i}^{2}$ on $\mathbb{R}_{++}^{m}$. Hence, $\mathbf{R}^{1}$ and $\mathbf{R}^{2}$ coincide on $\mathbb{R}_{++}^{m}$. Continuity of preferences, which is implied by analycity, then implies that $\mathbf{R}^{1}$ and $\mathbf{R}^{2}$ coincide on $\mathbb{R}_{+}^{m}$. Consequently, Independence of Infeasible Alternatives is trivially satisfied.

Theorem 6 is our possibility theorem for social choice correspondences defined on a monotone analytic preference domain and a full-dimensional compact agenda domain when the set of alternatives is the nonnegative orthant of a multidimensional Euclidean space. 
Theorem 6. If $X=\mathbb{R}_{+}^{m}$ with $m \geq 2$, on any monotone analytic preference domain and any full-dimensional compact agenda domain, there exist social choice correspondences that satisfy Independence of Infeasible Alternatives, Strong Pareto, Anonymity, and Arrow's Choice Axiom.

Proof. The proof exactly parallels the proof of Theorem 2 with monotone analytic preferences that have no critical points used instead of spatial preferences and the reference to Lemma 1 replaced by an appeal to Lemma 2 .

Donaldson and Weymark (1988) assume that $X=\mathbb{R}_{+}^{m}$, the preference domain consists of all profiles of continuous preference orderings, and the agenda domain consists of all compact, comprehensive sets that have a nonempty interior. ${ }^{21}$ They show that the four Arrow choice-theoretic axioms are consistent with these domain restrictions. The example they use to establish their theorem is not anonymous and it is an open question whether Anonymity can be satisfied on their domains. Their set of alternatives is the same as ours and their agenda domain is a full-dimensional compact agenda domain. Theorem 6 shows that Anonymity can also be satisfied if their preference domain is further restricted to include only profiles of monotone analytic preferences with no critical points.

With private goods and selfish individuals, Anonymity is vacuous because it is not possible to swap preferences between two individuals if they are selfish. By defining individual preferences directly on private consumption, a strengthened anonymity axiom that is more appropriate for private goods can be formulated by requiring that if individual preferences on own consumption are permuted among selfish individuals, then the choice on any agenda is unaffected. Note that if these preferences on own consumption are monotone, the induced preferences on the set of alternatives $X$ is also monotone, so the arguments that use monotonicity in this and the preceding section also apply to selfish individuals. As a consequence, a version of Theorem 6 using the strengthened form of Anonymity holds for private goods and selfish individuals if the set of admissible preferences for own consumption of each individual is a subset of $\mathcal{M}$.

\section{Concluding Remarks}

In our possibility theorem for monotone analytic preferences, each preference has no critical point. In our possibility theorem for Euclidean spatial preferences, each preference has one critical point. We conjecture that our two possibility theorems are special cases of a more general possibility theorem for preference domains that include only profiles of analytic preferences that have at most one critical point. The basis for our conjecture is our belief that the result of Debreu (1972) used to establish our ordinal version of the Analytic Continuation Principle can be generalized. Specifically, we conjecture that an analytic function with at most one critical point defined on $\mathbb{R}_{++}^{m}$ is completely characterized (up to a monotone transformation) by its gradient

\footnotetext{
${ }^{21}$ Their theorem also holds with additional restrictions on the admissible profiles and on the admissible agendas.
} 
function on an open subset of $\mathbb{R}_{++}^{m}$ when $m \geq 2$. As an example in Wagneur (1979) illustrates, an analytic function need not be characterized by its gradient function on an open subset of $\mathbb{R}_{++}^{m}$ if there are two or more critical points.

Our theorems, and those of Donaldson and Weymark (1988) and Gibbard, Hylland, and Weymark (1987), demonstrate that it makes a great deal of difference when working with restricted domains whether one is dealing with a social welfare function or a social choice correspondence. Preference domains that result in an impossibility theorem using a social welfare function may well yield a possibility theorem using a social choice correspondence when combined with an appropriate agenda domain. It is standard practice for general equilibrium theorists and welfare economists to place a priori restrictions on both preferences and the set of feasible alternatives. For example, assumptions are often made on production technologies and on endowments to ensure that the set of feasible alternatives is compact, comprehensive, and convex. Our results suggest that pursuing a similar strategy in social choice theory may be quite promising, sometimes yielding possibility theorems instead of impossibilities. ${ }^{22}$

\section{References}

Arrow KJ (1959) Rational choice functions and orderings. Economica 26: 121-127. Arrow KJ (1963) Social choice and individual values (2nd ed). John Wiley and Sons, New York.

Bailey MJ (1979) The possibility of rational social choice in an economy. Journal of Political Economy 87: 37-56.

Black D (1948) On the rationale of group decision-making. Journal of Political Economy 56: 23-34.

Blau JH (1957) The existence of social welfare functions. Econometrica 25: 302-313.

Border KC (1983) Social welfare functions for economic environments with or without the Pareto principle. Journal of Economic Theory 29: 205-216.

Border KC (1984) An impossibility theorem for spatial models. Public Choice 43: 293-305.

Bossert W, Weymark JA (1996) Utility in social choice. Forthcoming in: Barberà S, Hammond PJ, Seidel C (eds) Handbook of utility theory, Volume 2: Extensions. Kluwer Academic Publishers, Boston.

Campbell DE (1993) Euclidean individual preference and continuous social preference. European Journal of Political Economy 9: 541-550.

d'Aspremont C (1985) Axioms for social welfare orderings. In: Hurwicz L, Schmeidler D, Sonnenschein H (eds) Social goals and social organizations: Essays in memory of Elisha Pazner. Cambridge University Press, Cambridge, pp. 19-76.

Debreu G (1972) Smooth preferences. Econometrica 40: 603-615.

Debreu G (1976) Smooth preferences: A corrigendum. Econometrica 44: 831-832.

Dieudonné J (1969) Foundations of modern analysis. Academic Press, New York.

Dieudonné J (1971) Infinitesimal calculus. Hermann, Paris and Houghton Mifflin, Boston.

\footnotetext{
${ }^{22}$ Since this article was completed, Ehlers and Storcken (2001) have established a version of Theorem 1 in which the set of alternatives is a multidimensional compact convex subset of $\mathbb{R}^{m}$.
} 
Duggan J (1996) Arrow's theorem in public goods environments with convex technologies. Journal of Economic Theory 68: 303-318.

Donaldson D, Weymark JA (1988) Social choice in economic environments. Journal of Economic Theory 46: 291-308.

Ehlers L, Storcken T (2001) Arrow's theorem in spatial environments, unpublished manuscript, Department of Quantitative Economics, Maastricht University.

Enelow JM, Hinich MJ (1984) The spatial theory of voting: An introduction. Cambridge University Press, Cambridge.

Gibbard A, Hylland A, Weymark JA (1987) Arrow's theorem with a fixed feasible alternative. Social Choice and Welfare 4: 105-115.

Hansson B (1968) Choice structures and preference relations. Synthese 18: 443-458.

Kalai E, Muller E, Satterthwaite, M (1979) Social welfare functions when preferences are convex, strictly monotonic, and continuous. Public Choice 34: 87-97.

Kannai Y (1970) Continuity properties of the core of a market. Econometrica 38: $791-815$.

Kannai Y (1974) Approximation of convex preferences. Journal of Mathematical Economics 1: 101-106.

Le Breton M (1997) Arrovian social choice on economic domains. In: Arrow KJ, Sen A, Suzumura K (eds.) Social choice re-examined, Volume 1. Macmillan, London, pp. 72-96.

Le Breton M, Weymark JA (1996) An introduction to social welfare functions on economic and political domains. In: Schofield N (ed.) Collective decisionmaking: Social choice and political economy. Kluwer Academic Publishers, Boston, pp. 25-61.

Le Breton M, Weymark JA (2001) Arrovian social choice theory on economic domains. Forthcoming in: Arrow KJ, Sen AK, Suzumura K (eds.) Handbook of social choice and welfare, Volume 2. North-Holland, Amsterdam.

Maskin ES (1976) Social welfare functions for economics. Unpublished manuscript. Department of Economics, Harvard University and Darwin College, Cambridge University.

Ordeshook PC (1986) Game theory and political science: An introduction. Cambridge University Press, Cambridge.

Redekop J (1993a) Arrow-inconsistent domains. Social Choice and Welfare 10: 107126.

Redekop J (1993b) Social welfare functions on parametric domains. Social Choice and Welfare 10: 127-148.

Redekop J (1993c) The questionnaire topology on some spaces of economic preferences. Journal of Mathematical Economics 22: 479-494.

Sen AK (1979) Personal utilities and public judgements: Or what's wrong with welfare economics? Economic Journal 89: 537-558.

Wagneur E (1979) Saturation locale et concepts primitifs de la theorie du consommateur. Econometrica 47: 1549-1553. 


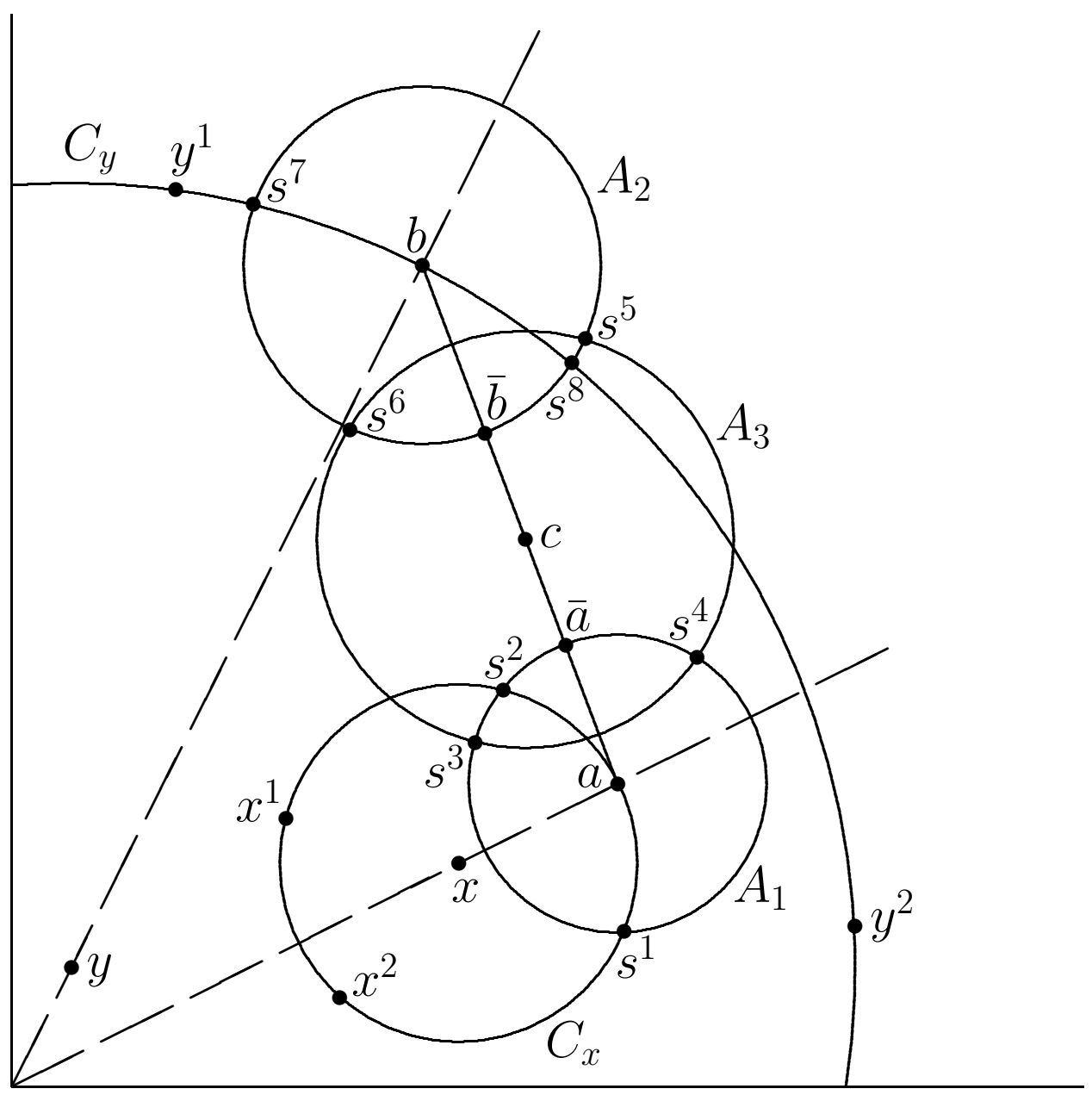

Figure 1 